\title{
ANÁLISE DOS IMPACTOS ESPERADOS E NÃO-ESPERADOS DA TAXA DE JUROS, CÂMBIO E INFLAÇÃO NO MERCADO BRASILEIRO
}

\author{
ANALYSIS OF EXPECTED AND NON-EXPECTED \\ IMPACTS OF INTEREST RATE, EXCHANGE RATE AND \\ INFLATION IN BRAZILIAN MARKET
}

Recebido 01/04/2011

Aceito $02 / 05 / 2012$

Marcelo Brutti Righi ${ }^{1}$

Sérgio Guilherme Schlender ${ }^{2}$

Paulo Sérgio Ceretta ${ }^{3}$

\section{RESUMO}

O presente trabalho procurou verificar os impactos esperados e não-esperados da taxa de juros, câmbio e inflação no mercado acionário brasileiro. Para tanto, aplicou-se modelo ARIMA para estimar os valores esperados das primeiras diferenças destas variáveis, bem como suas inovações em uma amostra de 383 observações semanais no período compreendido entre 02/03/2003 a 19/12/2007. Por fim, os resultados indicam que apenas os choques inesperados da taxa de câmbio no retorno das ações apresentaram significância.

Palavras-chave: Variáveis Macroeconômicas; ARIMA; Impactos Esperados e Não-Esperados.

\footnotetext{
${ }^{1}$ Mestrando em Administração pela Universidade Federal de Santa Maria (UFSM), Santa Maria, Rio Grande do Sul, Brasil. E-mail: marcelobrutti@hotmail.com

${ }^{2}$ Bacharel em Administração pela Universidade Federal de Santa Maria (UFSM), Santa Maria, Rio Grande do Sul, Brasil. E-mail: sergio.schlender1@gmail.com

${ }^{3}$ Doutor em Engenharia da Produção pela Universidade Federal de Santa Catarina (UFSC). Professor do Departamento de Ciências Administrativas da Universidade Federal de Santa Maria (UFSM), Santa Maria, Rio Grande do Sul, Brasil. E-mail: ceretta10@ gmail.com
} 


\section{ABSTRACT}

This paper examined the expected and non-expected impacts for interest rates, exchange and inflation rates in the Brazilian market. For this purpose, was applied the ARIMA model to estimate the expected value of the first differences of these variables, under a sample of 383 weekly observations for the period from 02/03/2003 to 19/12/2007. Finally, the results indicate that only unexpected shocks in the exchange rate on stock returns were significant.

Keywords: macroeconomic variables; ARIMA; expected and non-expected impacts.

\section{INTRODUÇÃO}

A década de 2000 foi caracterizada pelo forte desenvolvimento econômico-financeiro e constante valorização de investidores domésticos e externos em relação ao mercado de capitais brasileiro. Um cenário contrastante com aquele apresentado em períodos anteriores, no qual alguns elementos afetavam negativamente o comportamento de investidores, tais como: i) alto grau de concentração do mercado acionário, reduzido número de ações negociadas, ii) valorização acentuada de títulos públicos em detrimento aos títulos privados, e, sobretudo, iii) alta incerteza nas informações presentes nas ações (NOBREGA et al., 2000; NUNES et al., 2005).

Valorizando este novo cenário, transcendente às efêmeras especulações vinculadas aos fluxos de capitais, é percebido o fortalecimento da proteção dos investidores contra riscos indesejados (hedge) e o aumento na possibilidade desses investidores maximizarem suas respectivas carteiras de investimentos acionários (SILVA, SANTO e SILVA, 2003).

Mais do que isso, é factível refletir que esse contexto não se restrinja exclusivamente a elementos microeconômicos. Não menos importantes, alguns elementos macroeconômicos como inflação, câmbio e taxa de juros, apresentam características que condicionam o mercado acionário, em destaque no que tange ao comportamento dos investidores frente aos retornos e riscos esperados e inesperados das ações, oriundos de elementos dos próprios investimentos e variações inesperadas e adversas, nas quais incluem elementos macroeconômicos (FLOROS, 2004; SARI e SOYTAS, 2005; NUNES et al., 2005; DAMODARAN, 2007).

Atrelado a este comportamento, na existência de retornos de um investimento oriundo de informações previstas e não previstas, investidores acompanham a divulgação de dados macroeconômicos. Isso se deve a dois pontos importantes: i) a tomada de decisões que eliminem o risco esperado, por meio da diversificação de uma possível carteira na qual outros investimentos são aplicados, fortalecendo o retorno na carteira do investidor; e ii) a adoção de políticas de proteção, com apoio de modelos financeiros como o Capital Asset Pricing Model (CAPM) e a Arbitrage Pricing Theory (APT) (DHANKAR e ESQ, 2005), cujo objetivo é de encontrar riscos inesperados, que uma vez eliminados facilitam a obtenção de um retorno sistemático do investimento (ARAY, 2008; JORDAN, ROSS E WESTERFIELD, 2002; SHARPE, 1964; FAMA, 1970).

Diante do exposto, o presente estudo teve como objetivo verificar o impacto das: i) variações esperadas e ii) variações não esperadas das variáveis macroeconômicas sobre os retornos diários das ações brasileiras - extraídas no banco de dados da Bolsa de Mercadorias, Valores e Futuros do Brasil (BM\&FBovespa). Para tanto, será aplicado o modelo ARIMA (Auto Regressive Integrated Moving Average), respeitada as condições de estacionariedade das seguintes cotações semanais: i) taxa de câmbio comercial média semanal na relação das moedas Real e Dólar, representando a variável macroeconômica câmbio; ii) Índice Geral de Preços ao Consumidor Semanal (IPC-S), relacionado a inflação; iii) Taxa SELIC, atrelada a taxa de juros; e iv) Índice Boves- 
pa, que representa as cotações semanais do mercado financeiro. Não obstante, foram retirados 383 observações no período compreendido entre 02/03/2003 a 19/12/2007, correspondente ao mandato do último governo do país.

Além dessa Introdução, o presente trabalho será estruturado da seguinte forma: a seção 2 explanará sobre cada uma das variáveis macroeconômicas em estudo e respectivos efeitos nos retornos acionários; na seção 3, serão tomados aspectos metodológicos desta pesquisa, expondo a natureza dos dados utilizados, bem como o modelo econométrico, de acordo com o objetivo proposto; na seção 4, será apresentada a análise dos resultados obtidos, e na ultima seção, serão expostos as conclusões do presente estudo.

\section{REVISÃO DE LITERATURA}

\subsection{Inflação}

A relação entre preços acionários e taxa de inflação já é amplamente estudada pela literatura financeira, em especial no efeito dessa variável macroeconômica nos retornos de ativos do mercado financeiro. Estudos como os de Fama e Schwert (1977); Fama (1981); Nelson (1976); Geske e Roll (1983); Feldstein (1980); Pindyck (1984); Bakshi e Chen (1996); e Zhao (1999), constataram uma relação negativa entre retorno dos preços acionários e inflação, decorrente do que Fama (1981) apresentou como "hipótese de proxy effect" (NUNES, COSTA JR e MEURER, 2005). Tal hipótese apresenta o retorno dos preços como uma proxy da relação entre inflação e nível de atividade econômica: quando forte, o nível de atividade econômica impacta na taxa de inflação e no comportamento de investidores, que adotam políticas contra-cíclicas para o hedge de suas ações (MERIKAS e MERIKA, 2006).

Com isso, os retornos dos preços negociados no mercado financeiro tendem a ter um comportamento inverso (negativo) aos das taxas de inflação, atrelado à possibilidade de que os efeitos esperados da adoção de uma dessas políticas contra-cíclicas sejam superiores ao retorno esperado, decorrente do aumento no nível de atividade econômica. Dessa forma, a informação de uma possível melhoria na atividade econômica é prevista e incorporada aos preços de ativos de períodos anteriores, e ratifica o comportamento entre as variáveis (NUNES, COSTA JR e MEURER, 2005).

Não obstante, adaptando ao modelo proposto por Fisher (1930), em especial da composição da taxa de retorno esperado, Gutelkin (1983) apresenta a independência das variáveis componentes dessa taxa (retorno dos mercados esperado e taxas de inflação esperada). Com isso, investidores poderiam ser compensados por essas variações na compra de ativos (ver mais detalhes do modelo em NUNES, COSTA JR, MEURER e 2005).

Por fim, Caporale e Jung (1997) estudaram não apenas os efeitos esperados pela inflação, como também oriundos de variações (choques) não previstos pelos investidores, e constataram que os efeitos não-esperados da inflação se prolongam além do período em que as ações dariam cobertura aos impactos inflacionários. Mais do que isso, deve-se atentar a natureza do choque atrelado à inflação, já que conforme estudo de Hess e Lee (1999), o sinal de correlação positiva, por exemplo, pode dar um efeito positivo sobre os preços e a inflação (FLOROS, 2004).

\subsection{Taxa de Juros}

A taxa de juros também apresenta seu impacto na relação com os preços acionários. Muitos estudiosos, não satisfeitos com a hipótese de Fama (1981), adicionaram o elemento governamental e 
das políticas monetárias no escopo, ampliando a respectiva hipótese para o que Geske e Roll (1983) denominaram de hipótese da causalidade reversa. Esta hipótese determina uma inversão na expectativa do investidor quanto às variações da inflação, nas quais um conjunto de eventos macroeconômicos, além da inflação, estaria associado, por exemplo, a política monetária atrelada à taxa de juros (ver modelo de SOLNIK (1983) em NUNES, COSTA JR e MEURER, 2005).

Como exemplo (ver ROGERS, 2006), o aumento na taxa de juros doméstica elevaria o preço do prêmio que investidores estariam dispostos a pagar no mercado financeiro. Com aumento na taxa de juros, o governo eleva o valor dos títulos públicos para maiores remunerações, concorrendo com as remunerações do mercado financeiro, além de enfraquecer o consumo e os investimentos aplicados, desestimulando o mercado de capitais.

Com isso, retornos de ações estão negativamente relacionados com a taxa de juros, valendo-se as mesmas situações com as ocorridas com a inflação esperada (HANSEN e SINGLETON, 1983; CAMPBELL, 1993; MEDEIROS e RAMOS, 2004a e 2004b; RIGOBON e SACK, 2001).

\subsection{Câmbio}

Ao ser estudada pela literatura financeira, com destaques a Dornbush e Fischer (1980), Ajayi et al. (1998), Lee e Solt (2001), Mukherjee e Naka (1995), apresentaram uma relação positiva entre os retornos das ações e a taxa de câmbios (ARAUJO BASTOS, 2008; GROPPO, 2004). No entanto, não há relações em longo prazo entre essas duas variáveis.

Na hipótese de ocorrer um impacto esperado da taxa de câmbio nos retornos das ações, a explicação mais plausível é encontrada por Dornbush e Fisher (1980), em que a elevação dos preços externos e da competitividade das empresas nacionais no mercado externo, refletiria na valorização das ações, e, assim, possibilitando maior retorno (ARAUJO E BASTOS, 2008).

Por outro lado, a ocorrência de efeitos não-esperados da taxa de câmbio sobre os retornos acionários é sustentada pelo estudo de arbitragem de Ross, Westerfield e Jaffe (1995), baseado no respectivo modelo Arbitrage Pricing Theory (APT) de Ross (1976). Tal estudo afirma que numa operação compensatória de compra e venda, certo agente, verificando condições favoráveis (entre elas os efeitos de variáveis macroeconômicas como câmbio), efetua a compra de um negócio no mercado mais barato e a venda deste negócio no mercado mais caro, sem risco e com retorno garantido, já que títulos externos e internos são substitutos entre si.

No entanto, essa igualdade de preço que possibilita a arbitragem, quando não sustentada, pode gerar efeitos contrários aos encontrados por Dornbush e Fisher (1980), atrelados diretamente a barreiras comerciais, informação imperfeita, aversão ao risco, além da presença monopolística ou oligopolista, monopsônica ou oligopsônica, em um ou mais dos mercados, decorrente das peculiaridades desses mercados (SEXTON et al.,1991; NOGUEIRA et al., 2005). Com isso, é possível existir uma inversão na relação entre taxa de câmbio e retorno das ações.

Não obstante, em relação às peculiaridades dos mecados, dá-se destaque ao estudo de causalidade de Granger et al. (1998), que avaliando as relações de causalidade entre retornos das ações de diversos mercados asiáticos e a taxa de câmbio, com base no modelo de Ajayi et al. (1998), constatou que os retornos acionários determinam a taxa de câmbio em economias desenvolvidas, enquanto que em economias emergentes o mesmo não acontecia, justificada pela falta de consistência na relação entre as variáveis, sobretudo a estrutura do mercado financeiro estudado: países emergentes tem mercado financeiro menor, mais concentrado e menos acessível aos investidores. 


\section{MÉTODO}

Visando estimar os impactos dos valores esperados e não esperados do Câmbio, Inflação e Taxa de Juros no mercado de capitais brasileiro, foram coletadas cotações semanais da relação Dólar/Real (Câmbio), IGP-S (Inflação), Selic (Taxa de Juros) e Índice Bovespa (Ibovespa), que representa o mercado, no período de 02/03/2003 a 19/12/2007, totalizando uma amostra composta por 383 observações. A escolha destas datas se justifica por representarem o governo da atual situação de governo do país, excetuando os meses iniciais devido a instabilidade causada pela mudança de gestão no país.

Primeiramente foi realizado teste de raiz unitária Dickey-Fuller Aumentado (ADF), de Dickey e Fuller (1981), nas séries contidas no estudo, com o intuito de verificar a condição de estacionariedade dos dados. Assim, as variáveis que apresentaram raiz unitária forma diferenciadas, a fim de apresentar estacionariedade. Posteriormente, almejando estimar os valores esperados e não esperados das variáveis macroeconômicas Câmbio, Inflação e Juros, foram estimados modelos ARIMA, que segundo Tsay (2010) basicamente, combina as ideias dos modelos auto regressivos (AR) e média móvel (MA) em uma forma compacta, de tal maneira que o número de parâmetros permaneça reduzido. Um modelo $\operatorname{ARIMA}(p, d, q)$ genérico tem a seguinte forma, conforme exposto na formulação [1].

$$
\Delta X_{t}=\varphi_{0}+\sum_{i=1}^{p} \varphi_{i} \Delta X_{t-i}+a_{\tau}+\sum_{i=1}^{q} \theta_{i} a_{t-i}
$$

Onde ${ }^{X_{t}}$ é a série temporal a ser ajustada pelo modelo; $\Delta$ é o operador de diferença usualmente utilizado; $\varphi_{i}$ e $\theta_{i}$ são parâmetros; $\left(a_{t}\right)$ é uma série ruído branca; $p$ e $q$ são inteiros não-negativos correspondentes ao número de defasagens das partes AR e MA; $d$ é o número de vezes que a série $X_{t}$ precisou ser diferenciada para obter a condição de estacionariedade.

Desta forma, de posse dos valores esperados, bem como as inovações obtidas por meio da modelagem ARIMA, foi estimada a regressão das cotações do mercado contra os valores esperados e não esperados das variáveis macroeconômicas. A equação resultante possui sua formulação matemática expressa em [2].

$$
M_{t}=\beta_{0}+\beta_{1} C_{t}+\beta_{2} a_{c, t}+\beta_{3} I_{t}+\beta_{4} a_{i, t}+\beta_{5} J_{t}+B_{6} a_{j, t}+\varepsilon_{t}
$$

Em [2], $\boldsymbol{M}_{\boldsymbol{t}}, \boldsymbol{C}_{\boldsymbol{t}}, I_{\boldsymbol{t}}, \boldsymbol{I}_{\boldsymbol{t}}$, são os valores esperados do Mercado, Câmbio, Inflação e Taxa de Juros no instante $t$, respectivamente; $\boldsymbol{a}_{\boldsymbol{c}, \boldsymbol{t}}, \boldsymbol{a}_{\boldsymbol{i}, \boldsymbol{t}}, \boldsymbol{a}_{j, t}$, são os choques (não esperados) do Câmbio, Inflação e Taxa de Juros no instante $t$, respectivamente; $\beta_{k}, k=1, \ldots, 6$, são parâmetros de regressão; $\varepsilon_{t}$ é o erro do modelo no instante $t$.

\section{ANÁLISE DOS RESULTADOS}

Inicialmente, foi realizado teste ADF nas séries temporais das variáveis citadas na parte metodológica deste estudo, almejando identificar a condição de estacionariedade nas mesmas. Com exceção da Inflação, nenhuma das variáveis foi capaz de rejeitar a hipótese nula de raiz unitária, e assim não-estacionariedade. Após, foram calculadas as primeiras diferenças dos logaritmos das variáveis que possuem raiz unitária. Tais séries rejeitaram ao nível crítico de $5 \%$ a hipótese nula do teste ADF de não-estacionariedade. Os valores dos testes ADF aplicados, assim como sua significância, estão contidos na Tabela 1. 
Tabela 1. Valores calculados dos testes ADF utilizados e seus P-valores.

\begin{tabular}{llll}
\hline \multicolumn{1}{c}{ Variável } & ADF & P-valor & \\
\hline Nível & & & \\
\hline Ibovespa & $-1,1344$ & 0,7044 & \\
Câmbio & $-2,8411$ & 0,0526 & $*$ \\
Inflação & $-6,0790$ & 0,0000 & $*$ \\
Taxa de juros & $-2,8011$ & 0,0581 & \\
\hline Primeira Diferença & & & \\
\hline Ibovespa & $-15,2393$ & 0,0000 & $*$ \\
Câmbio & $-12,7786$ & 0,0000 & $*$ \\
Taxa de Juros & $-13,8271$ & 0,0000 & $*$ \\
\hline *
\end{tabular}

* Significantemente diferente de zero ao nível de $5 \%$.

Em seguida, visando obter os valores esperados e não esperados de Câmbio, Inflação e Taxa de Juros, foram estimados modelos ARIMA $(1,1,1)$ para estas variáveis (para Inflação foi estimado o modelo ARMA $(1,1)$ devido a não precisar ser diferenciada para obter estacionariedade). Os resultados dos parâmetros estimados para estes modelos estão presentes na Tabela 2.

Os resultados da Tabela 2 evidenciam que, os modelos lineares estimados para Câmbio e Inflação possuem coeficientes auto-regressivos significantes. Este fato indica que ambas as variáveis são influenciadas por seu período anterior de negociação. Não obstante, o modelo da variável Inflação apresentou ainda parâmetro de média móvel significativo. Já o modelo da Taxa de Juros não exibiu nenhum parâmetro significativo. Isto pode ser explicado, em grande parte, pela falta de variação existente na série de valores desta variável, já que é alterada ou mantida com base em decisões governamentais (exemplo a ser visto em Rogers, 2006).

Tabela 2. Coeficiente dos modelos ARIMA estimados para as diferenças dos logaritmos do Câmbio e Taxa de juros e ARMA para a variável Inflação.

\begin{tabular}{|c|c|c|c|c|c|}
\hline Parâmetro & Coeficiente & Erro Padrão & Teste $t$ & Significância & \\
\hline \multicolumn{6}{|l|}{ Câmbio } \\
\hline Constante & $-0,0044$ & 0,0023 & $-1,9219$ & 0,0546 & \multirow{3}{*}{ * } \\
\hline$A R$ & $-0,6354$ & 0,2441 & $-2,6031$ & 0,0092 & \\
\hline MA & 0,5223 & 0,2697 & 1,9367 & 0,0527 & \\
\hline \multicolumn{6}{|l|}{ Inflação } \\
\hline Constante & 0,5438 & 0,1148 & 4,7336 & 0,0000 & * \\
\hline$A R$ & 0,9294 & 0,0223 & 41,7138 & 0,0000 & * \\
\hline MA & 0,3400 & 0,0376 & 9,0295 & 0,0000 & * \\
\hline \multicolumn{6}{|l|}{ Taxa de Juros } \\
\hline Constante & $-0,0397$ & 0,0143 & $-2,7706$ & 0,00560 & * \\
\hline$A R$ & 0,1194 & 0,7073 & 0,1689 & 0,86591 & \\
\hline MA & $-0,1379$ & 0,7034 & $-0,1961$ & 0,84453 & \\
\hline
\end{tabular}

* Significantemente diferente de zero ao nível de $5 \%$.

Entretanto, não é a estimação e análise destes modelos lineares o escopo fundamental desta pesquisa, sendo que os mesmos possuíram a serventia de fornecer os valores esperados das variáveis, bem como os resíduos (valores não-esperados) das variáveis em questão.

Desta forma, de posse dos valores esperados e não-esperados de Câmbio, Inflação e Taxa de Juros, bem como da variável proxy do mercado de capitais brasileiros, o índice Bovespa, foi possível estimar a equação proposta em [2]. Os valores obtidos são exibidos na Tabela 3.

Com base nos resultados expostos na Tabela 3, podemos concluir que apenas as inovações (valores não-esperados) da variável Câmbio afetam significativamente os log-retornos do mercado de capitais brasileiros. Tal impacto se dá em sentido negativo, o que indica que variações inesperadas na relação Dólar/Real implicam em variações negativas no nível de pontos do Índice Bovespa. Isso pode estar atrelado à assimetrias de informação, inversão a risco ou à aspectos estruturais dos mercados que possibilita esse tipo de relacionamento (ver SEXTON et al. ,1991; NOGUEIRA et al., 2005) 
Tabela 3. Parâmetros da regressão do retorno do mercado contra os valores esperados e não esperados de Câmbio, Inflação e Taxa de Juros.

\begin{tabular}{lccrl}
\hline \multicolumn{1}{c}{ Parâmetro } & Coeficiente & Erro Padrão & Teste $\boldsymbol{t}$ & p-valor \\
\hline Constante & $-0,0051$ & 0,0097 & $-0,5273$ & 0,5983 \\
Câmbio esperado & 0,0091 & 0,0068 & 1,3451 & 0,1794 \\
Câmbio não-esperado & $-0,3806$ & 0,0406 & $-9,3647$ & 0,0000 \\
Inflação esperada & $-0,0039$ & 0,0058 & $-0,6672$ & 0,5050 \\
Inflação não esperada & $-0,0159$ & 0,0168 & $-0,9427$ & 0,3465 \\
Taxa de Juros esperada & $-0,0006$ & 0,0007 & $-0,9053$ & 0,3658 \\
Taxa de Juros não-esperada & $-0,0069$ & 0,0070 & $-0,9867$ & 0,3244 \\
\hline
\end{tabular}

* Significantemente diferente de zero ao nível de $5 \%$.

Complementando os resultados obtidos para a equação [2] e visando dar robustez ao modelo utilizado, são apresentadas na Tabela 4 estatísticas de diagnóstico do mesmo.

Tabela 4. Estatísticas de diagnóstico da regressão do retorno do mercado contra os valores esperados e não esperados de Câmbio, Inflação e Taxa de Juros.

\begin{tabular}{lc}
\hline Estatística & Valor \\
\hline R-quadrado & 0,2020 \\
R-quadrado ajustado & 0,1893 \\
F(6, 377) & 15,9076 \\
P-valor(F) & 0,0000 \\
\hline
\end{tabular}

Os diagnósticos do modelo [2], expostos na tabela 4, indicam que as variáveis macroeconômicas e suas inovações explicam cerca de $20 \%$ das variações do retorno do mercado em torno de sua média. Considerando-se uma ponderação pelos graus de liberdade, o modelo obtém um poder de explicação de $18,9 \%$ das variações da variável dependente. Verifica-se ainda, que o modelo é significativo no geral, pois apresentou uma estatística $F$ estatisticamente diferente de zero, com P-valor = 0,0000 . Estes resultados indicam bom ajuste por parte da equação [2] estimada.

\section{CONCLUSÃO}

O presente artigo teve como objetivo a observação do impacto das variáveis macroeconômicas nos retornos diários esperados e inesperados das ações brasileiras. Para tanto, foram selecionados as variáveis Câmbio, Inflação e Taxa de Juros para realizar o impacto destas no retorno esperado e inesperado dos preços de cotação semanal do Índice Bovespa, num total de 383 observações no período compreendido entre 02/03/2003 a 19/12/2007.

Aplicado o método ARIMA, foi constatado que apenas as variáveis Câmbio e Inflação recebem influência de período defasado. Com a variável Taxa de Juros foi observado uma inexistência de parâmetros significativos, decorrente da natureza governamental das decisões de alterações e constância das taxas.

Atentando aos efeitos esperados e não-esperados dessas variáveis no retorno acionário, foi verificado significância apenas nos choques inesperados da variável Câmbio. Tal choque apresenta relação negativa no retorno dos preços das ações, indicando que as variações inesperadas da taxa cambial afetam negativamente o retorno dos ativos financeiros avaliados. Isso se deve a dificuldade no uso de arbitragem em ativos de dois mercados diferentes, decorrentes de barreiras comerciais, informação imperfeita, aversão ao risco e aspectos estruturais do mercado em questão (SEXTON et al. ,1991; NOGUEIRA et al., 2005). Além disso, as variáveis macroeconômicas, com ponderação nos graus de liberdade e significância na estatística $F$, obtiveram grau de explicação de $18,9 \%$ das variações no retorno das ações.

Por fim, sugere-se que, em futuros estudos acerca do tema, sejam estabelecidos estudos com outras variáveis macroeconômicas domésticas e externas, para dar maior resolução aos efeitos esperados e não-esperados das variáveis correspondentes no retorno das ações. 


\section{REFERÊNCIAS}

AJAYI, R. A.; FRIEDMAN, J.; MEHDIAN, S. M. On the relationship between stock returns and exchange rates: Test of Granger causality. Global Finance Journal, v. 2, n.9, p. 241251,1998.

ARAUJO, E.; BASTOS, F.A.S. Relações entre retornos acionários, juros, atividade econômica e inflação. Brazilian Business Review, v. 5, n.1 p. 51-73. 2008.

ARAY, $H$. Effects of macroeconomic announcements on stock returns across volatility regimes. The Papers from Department of Economic Theory and Economic History of the University of Granada. Dec/2008. Disponível em: http://www.ugr.es/ teoriahe/ RePEc/gra /wpaper/thepapers08_17.pdf Acessado em: 21/01/2011.

BAKSHI, G. S.; CHEN, Z. Inflation, Asset Prices, and the Term Structure of Interest Rates in Monetary Economies. Review of Financial Studies, v. 9, n.3, p. 241-75, 1996.

CAMPBELL, J. Y. Intertemporal asset pricing without consumption data. American Economic Review, v. 83, n.3, p. 487-512, 1993.

CAPORALE , T.; JUNG, C. Inflation and Real Stock Prices. Applied Financial Economics, v. 7, n. 4, p. 265-66, 1997.

DAMODARAN, A. Avaliação de empresas. 2 ed. São Paulo: Pearson Prentice Hall, 2007.

DHANKAR, R. S.; ESQ, R. Arbitrage Pricing Theory and the Capital Asset pricing Model: Evidence from the Indian Stock Market. Journal of Financial Management and Analysis, v.18, n.1, p.14-27, 2005.

DICKEY, D. A.; FULLER, W. Likelihood Ratio Statistics for Autoregressive Time Series with a Unit Root. Econometrica, v. 49, n.2, p. 10571072, 1981.

DORNBUSH, R.; FISCHER, S. Exchange rates and current account. American Economic Review, v. 70, n.5, p.960-971, 1980.

FAMA, E. F. Stock returns, real activity, inflation and money. American Economic Review, v. 71, n.4, p.545-565, 1981.

FAMA, E. F.; SCHWERT, G. Asset Returns and Inflation. Journal of Business, v. 55, n.3, p. 201231, 1977.

FAMA, E. F. Efficient capital markets: a review of theory and empirical evidence. The Journal of Finance, v. 25, n. 2, p. 383-417, 1970.

FELDSTEIN, M. Inflation and the stock market. American Economic Review, v. 71, n.4, p. 84947, 1980.

FISHER, I. The Theory of Interest. New York: Macmillan, 1930.

FLOROS, C. Stock Returns and Inflation in Greece. Applied Econometrics and International Development. v. 4, n.2, p. 55-68, 2004.

GESKE, R.; ROLL, R. The fiscal and monetary linkage between stock returns and inflation. Journal of Finance, 38(1):1-33, 1983.

GRANGER, C. W. J.; HUANG, B. N.; YANG, C. W. $A$ bivariate causality between stock prices and exchange rates: evidence from recent Asia FLU. Discussion Paper. Department of Economics. University of California, San Diego, 1998.

GROPPO, G. S. Causalidade das variáveis macroeconômicas sobre o lbovespa. Dissertação da Escola Superior Luiz de Queiroz, da Universidade de São Paulo (USP). Piracicaba, SP. 2004.

GUTELKIN, N. B. Stock markets returns and inflation: Evidence from the other countries. Journal of Finance, v. 38, n.1, p.49-65, 1983.

HANSEN, L. P.; SINGLETON, K. J. Stochastic consumption, risk aversion, and the temporal behavior of asset returns. Journal of Political Economy, v. 91, n.2, p.249-265, 1983. 
HESS, P. J.; LEE, B.S. Stock Returns and Inflation with Supply and Demand Shocks. Review of Financial Studies, v. 12 , n. 4, p. 1203-1218, 1999.

JORDAN, B. D.; ROSS, S. A.; WESTERFIELD, R. W. Princípios da Administração Financeira. 2 ed. São Paulo. Atlas, 2002.

LEE, W. Y.; SOLT, M. E. Economic exposure and hysteresis: Evidence from German, Japanese and U. S. stock markets. Global Finance Journal, v. 12, n.2, p.217-235, 2001.

MEDEIROS, O. R.; RAMOS, F. C. Determinantes do desempenho e volatilidade da Bovespa: um estudo empírico. Anais... IV Congresso de Controladoria e Contabilidade, São Paulo, FEA/ USP, 2004a.

MEDEIROS, O. R.; RAMOS, F. C. Evidências empíricas sobre o comportamento do mercado de ações no Brasil. Anais... IV Congresso Virtual de Administração, São Paulo, CONVIBRA, 2004b.

MERIKAS, A. G.; MERIKA, A. A. Stock prices response to real economic variables: the case of Germany. Managerial Finance, v. 32, n.5, p.446-450, 2006.

MUKHERJEE, T.; NAKA, A. Dynamic relations between macroeconomic variables and Japanese stock market: an application of a vector error correction model. The Journal of Financial Research, v.18, n.2, p.223-237, 1995.

NELSON, C. R. Inflation and Rates of Return on common Stocks. Journal of Finance, v. 31, n.5, p. 471-483, 1976.

NOBREGA, M., LOYOLA, G.; GUEDES FILHO, E.; PASQUAL, D. O mercado de capitais: sua importância para o desenvolvimento e os entraves com que se defronta no Brasil. Tendências - consultoria integrada Bovespa. São Paulo, 2000.

NOGUEIRA, F. T. P.; AGUIAR, D. R. D.; LIMA, J. E. Integração espacial no mercado brasileiro de café arábica. Nova Economia, v. 15, n. 2, p. 91112, 2005.

NUNES, M.S.; COSTA JR, N. C. A.; MEURER, R. A relação entre o mercado de ações e as variáveis macroeconômicas: uma análise econométrica para o Brasil. Revista Brasileira de Economia, v.59, n.4, p. 585-607, 2005.

PINDYCK, B. S. Risk, inflation, and the stock market. American Economic Review, v. 74, n.4, p. 335-351, 1984.

RIGOBON, R.; SACK, B. Measuring the reaction of monetary policy to the stock market. Cambridge: NBER - Working Papers n.8350, 32, 2001

ROGERS, P. Governança corporativa, mercado de capitais e crescimento econômico no Brasil. Dissertação. Faculdade de Gestão e Negócios da Universidade Federal de Uberlândia, 2006.

ROSS, S. A.; WESTERFIELD, R. W.; JAFFE, J. F. Administração financeira: corporate finance. São Paulo: Atlas, 1995.

ROSS, S. The arbitrage theory of capital pricing. Journal of Economic Theory, v.13, n.2, 112134, 1976.

SARI, R.; SOYTAS, U. Inflation, Stock Returns, and Real Activity in Turkey. The Empirical Economics Letters, v 6, n.2, p. 181-192, 2005.

SEXTON, R. J.; KLING, C. L.; CARMAN, H. F. Market integration, efficiency of arbitrage, and imperfect competition: methodology and application to U.S. celery. American Journal of Agricultural Economics, v. 73, n. 3, p. 569-580, 1991.

SHARPE, W. F. Capital asset prices: a theory of market equilibrium under conditions of risk. Journal of Finance, v. 19, n. 3, p. 35-10, 1964.

SILVA, W. V.; SANTO, E. L.; SILVA, L. S. C. Cointegração entre os preços da soja cotados nos mercados brasileiro e norte-americano: uma análise empírica. Caderno de Pesquisas em 
Administração, v. 10, n. 3, p. 69-78, 2003.

SOLNIK, B. The relations between stock prices and inflationary expectations: The international evidence. Journal of Finance, $v$. 38, n.1, p.35-48, 1983.

TSAY, R. S. Analysis of financial time series.2 ed. New Jersey: John Wiley \& Sons, 2010.

ZHAO, X. Q. Stock Prices, Inflation and Output: from China. Applied Economics Letters, v. 6, n.6, p. 509-521, 1999. 